\title{
An m-Health Monitoring System for Children with Suspected Arrhythmias
}

\author{
E. Kyriacou, C. Pattichis, M. Pattichis, A. Jossif, L. Paraskeva, A. Konstantinides, D. Vogiatzis
}

\begin{abstract}
Advances in wireless communications and networking technologies as well as computer and medical technologies, enable the development of small size, power efficient and more reliable medical multi-parameter recording systems, which can be used for continuous monitoring of patients. Through this paper we present the basic architecture and initial development steps of an m-Health monitoring system that will be used in order to monitor children with suspected cardiac arrhythmias. The proposed system will be based on sensor networks, in order to monitor a subject while being in a predefined area like his/her house; while a module based on PDAs and wearable ECG recorders will be used in order to extent the coverage outside the patient's house. The system will be based on a variable sampling rate to conserve power for the possible arrhythmia episode. The system design has been completed, the hardware specifications have been decided and currently the system is going through the development phase.
\end{abstract}

\section{INTRODUCTION}

$\mathrm{T}$ elemedicine has been used for many years in order to improve health care provision or for patient monitoring solutions. Several issues such as the computational capability, size of the devices, power efficiency and cost have been limiting the availability of devices and services to a few special cases [1]. Recently though, the current advancements in communications and computer systems can help us develop general-purpose systems that are more efficient, much smaller and at lower costs.

In this study, we will focus on the continuous monitoring of children with suspected cardiac arrhythmias in order to evaluate the size and severity of the problem. Arrhythmia is one of the most difficult problems in Cardiology both in

Manuscript received April 2, 2007. This work was supported in part by the Research Promotion Foundation of Cyprus under the Grant IPE/PLHRO/0506 "Wireless Microsensors for the Detection of Cardiac Arrythmias (AMEK)"

E. Kyriacou is with the Dep. of Computer Science, University of Cyprus and the Dep. of Comp. Science and Engineering, Frederick Insitute of Technology, Cyprus e-mail: ekyriac@cs.ucy.ac.cy.

C. Pattichis is with the Department of Computer Science, University of Cyprus, Cyprus (e-mail: pattichi@es.ucy.ac.cy).

M. Pattichis is with the Department of Electrical and Computer Engineering, University of New Mexico, NM, USA. (e-mail: pattichis@eece.unm.edu)..

A. Jossif is with the Makarios Hospital, Ministry of Health, Cyprus (e-mail: ajossif@cytanet.com.cy).

L. Paraskevas is with the Department of Computer Science, University of Cyprus, Cyprus (e-mail: Loucas.Paraskeva@gmail.com).

A. Konstantinides is with the Department of Computer Science, University of Cyprus, Cyprus (e-mail: angelos.constantinides@gmail.com).

D. Vogiatzis is with the Department of Computer Science, University of Cyprus, Cyprus (e-mail: dimitrv@cs.ucy.ac.cy). terms of diagnosis and management. The problem is particularly pronounced in Pediatric Cardiology because of the variety of etiologies and the difficulty that the children are having in trying to communicate their symptoms. For example in the case of hypertrophic cardiomyopathy, it is known that children are at higher risk for arrhythmias and sudden death than adults. In most of the cases an ECG tracing is required and this is sufficient for an accurate diagnosis, whereas in some cases, a more sophisticated modality is required [2], [3].

A relatively recently recognized entity, the Isolated Noncompaction of the Left Ventricle (NCLV), which is a rare form of cardiomyopathy, poses a special problem. A subset of patients with this disease are especially prone to arrhythmia and sudden death. It is not always possible to estimate the risk of each patient with the available test modalities even if we include genetic testing. The holter monitor has proved insufficient because it is limited to 24 or 48 hours of recording during which the patient may be asymptomatic. We care for a group of such children, some of whom are at imminent risk of sudden death [2], [3].

In this study, a mobile health (m-Health) system that will be able to monitor children continuously during their daily life activities is proposed. The system will be able to do realtime acquisition and transmission of ECG signals from the patient, and facilitate an alarm scheme able to identify possible arrhythmias so as to notify the on-call doctor and the relatives of the child that an event or something that denotes malfunction is happening. This system is a significant extension over our earlier telemedicine work in real-time ambulatory monitoring systems [4].

\section{EXAMPLE CASES}

In order to better appreciate the problem a brief description of two cases is presented.

\section{A. Case 1}

A few years ago we lost one such child, a four-year-old boy that was our first patient to be diagnosed with this disease. He was presented with a near miss episode of sudden death while at the nursery school and he was revived at the hospital where he was brought unconscious. He was then referred to UK for electrophysiological studies that were essentially normal. He had periods of bradycardia on the holter monitor and during his hospitalization. It was believed that the episode of loss of consciousness may have 


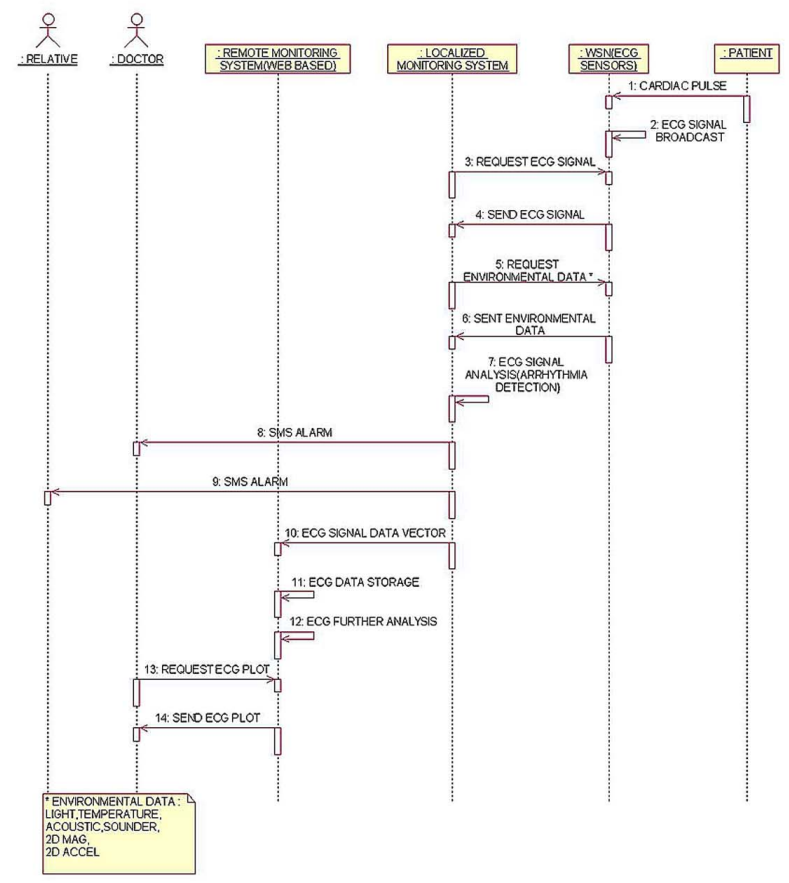

Figure 1. UML - sequence diagram of the actions being performed during the in-house case

been related to bradycardia and a pacemaker was implanted. A few months later, while at school, he lost consciousness again and was brought to the hospital but he could not be revived this time. Before dying, an ominous form of arrhythmia (torsades de pointe) was recorded. A few weeks before death, his mother reported a very short episode of near loss of consciousness that retrospectively could have been a short episode of arrhythmia but unfortunately there was no record of it.

\section{B. Case 2}

The second case is that of a nine year old girl with a history of several episodes of loss of consciousness and NCLV. In her case, we were lucky to record one such episode on the holter monitor. She had multiple episodes of supravenrticular tachycardia following one of which there was a prolonged pause that coincided with the witnessed episode of loss of consciousness. She was then started on beta blockers and she remains asymptomatic over the last three years.

\section{Motivation}

The description of these two cases highlights the wide range of clinical presentation and the variety of the underlying arrhythmias that these children present. Some of these children are high risk for sudden death and at the same time it is very difficult to decide for the proper treatment, making their ECG monitoring a very important task. The methods available today for ECG monitoring are clearly insufficient for this purpose. We need a noninvasive or minimally invasive way to record the ECG for extended periods of time and at the same time perform automatic analysis continuously or at frequent intervals.

\section{USER REQUIREMENTS AND SYSTEM DESIGN}

In general the problem has been divided into two cases. The first one, called "In-house case" the subject is located in his/her house. While for the second, called "Moving patient case" the subject might be located anywhere else. Our goal is the continuous 24 hours monitoring of the child.

\section{A. In-house case}

During this case, a sensor network installed in the child's house that will be used in order to continuously monitor ECG signals from the patient [5] - [8]. Several other environmental parameters like light, temperature, sound, acceleration will also be monitored so as to continuously check the living conditions. The ECG signal will be recorded by a sensor carried from the child, that will be part of a wireless sensor network (WSN) installed in the house. Signal information from the wearable sensor will be propagated to a local monitoring station which will also act as a gateway to the rest of the monitoring network. This case can be described as follows (see also Fig. 1):

a. The cardiac pulse is propagated through the WSN to the local monitoring station with an embedded broadcast algorithm.

b. The local monitoring station is responsible for collecting environmental measurements (e.g.temperature, 2D accelerometer, 2D magnetic field meter, sound, light):

- Sample the ECG signal.

- $\quad$ Store the sensor data locally.

- Analyze the ECG signal in order to detect possible cardiac arrhythmias.

c. In the case of a detected arrhythmia:

- Send an alarm message to the central monitoring station (located in hospital).

- Send an alarm via an SMS to the supervising doctor and a relative.

$\mathrm{d}$. The central monitoring station is responsible to:

- Store data sent from the local monitoring station.

- Display data transmitted from the local monitoring stations and through a web interface.

- Analyze the ECG signal further.

- Evaluate the alarm case and send a message (SMS, email etc.) to the doctor on-call.

\section{B. Moving patient case}

The second case is more general that will be used in order to complete the coverage of the system. For this case, the child will be monitored using the same ECG recording device but the signals will be transmitted, through a PDA device, directly to the central monitoring system. The transmission will be performed through the use of $2.5 \mathrm{G}$ and $3 \mathrm{G}$ mobile communication networks (GPRS/UMTS) [1]. 


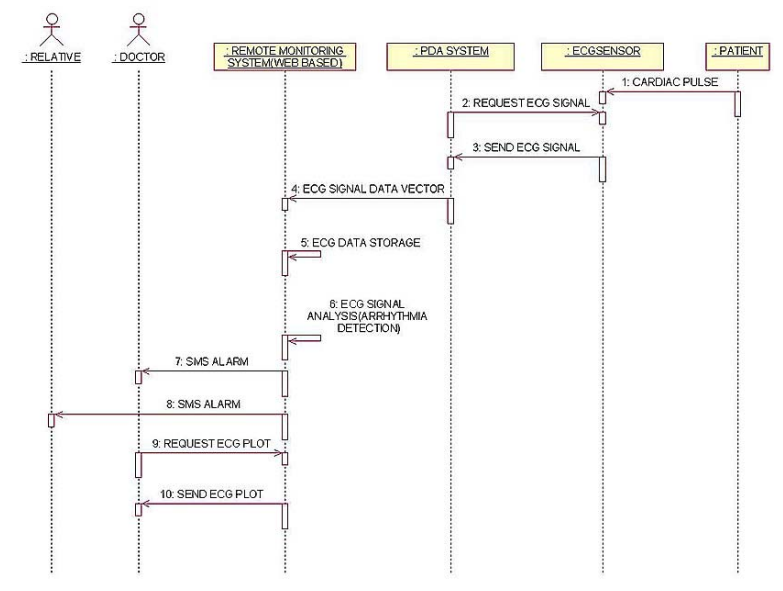

Figure 2. UML - sequence diagram of the actions performed for the Moving patient case

The information will be stored locally on the PDA and a basic analysis of the signals will be performed locally on the mobile unit (depending on the equipment specification). The central station will be responsible for the storage and analysis and display of information, as well as the notification of the on-call doctor and the relative in case of an alarm. The case can be described as (see also Fig. 2):

a. The cardiac pulse is propagated through the ECG sensors

to a PDA carried by the patient. The PDA system is responsible to:

- Sample the ECG signal and send it via GPRS/UMTS to the remote monitoring station.

b. The remote monitoring system is responsible to:

- Store data for the specific patient.

- Analyze the ECG signal in order to detect possible cardiac arrhythmias.

- Send an alarm via an SMS or e-mail to the supervising doctor and a relative.

- Display data locally and through the web.

\section{Variable rate ECG signal recording}

Holter monitoring is the most common case of ECG monitoring; but it can be applied for a short period of time (24-48 hours) and the processing can not be done in real time. 3-lead acquisition arrangements are the most common used for this case [9]. In general, monitoring of patients through an ECG signal can be performed through 1 to 12 leads signal acquisition; depending on the case and equipment available. Clearly though, this system is inappropriate for continuous-time monitoring due to significant limitations in the available power.

We propose the use of a variable-rate signal processing system that will be used to reduce the power requirements by reducing the sampling rate during normal operation, while saving the high sampling-rate and transmission during a possible arrhythmia session. To recognize the power savings, we note that power consumption is directly proportional to the frequency of operation. Thus, we can

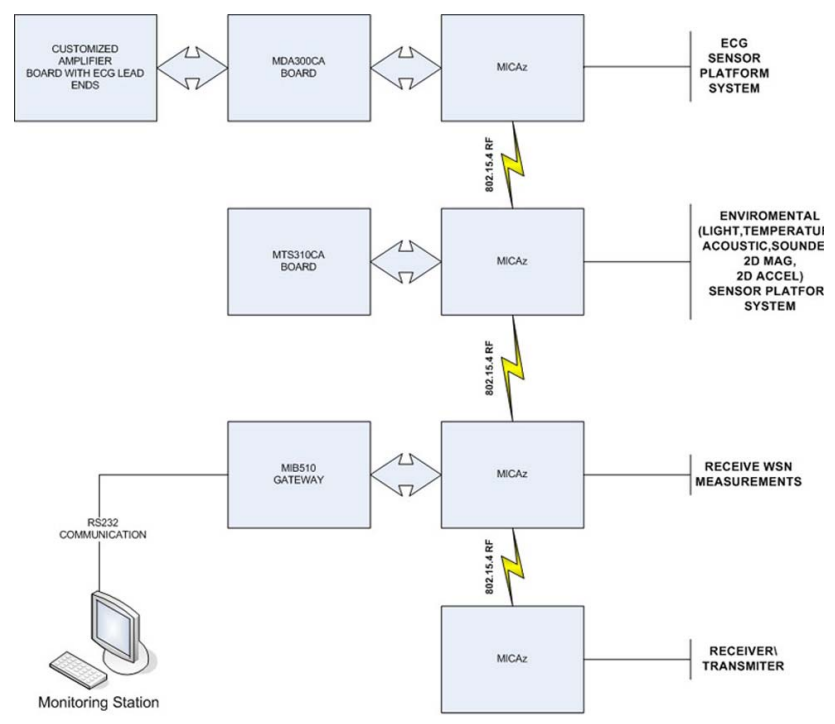

Figure 1. Block diagram of the in-house wireless sensor network

reduce power consumption by increasing the sampling period $T_{s}$. We can show that without changing the antialiasing analog filter prior to sampling, using a digital filter of variable bandwidth $B W$, we can produce a properly sampled ECG signal, sampled at $2(1-1 / B W) T_{S}$. Furthermore, larger sampling periods can be accommodated by varying the analog cutoff frequency of the anti-aliasing analog, lowpass filter. We had decided to have continuous transmission of one lead of ECG signal. For the case of a moving patient, three leads will be acquired and stored on the device, but only one waveform will be transmitted continuously [7].

\section{Sensor networks}

Research on sensor networks is relatively recent and is currently one of the emerging fields of information technology, combining sensing with communications so as to have continuous monitoring and support the mobility of sensors [8]. For our case we have chosen to develop a Motebased sensor network based on Crossbow ${ }^{\circledR}$ equipment [10]. In general, motes can operate using three types of topologies which are: 1) point to point topology, i.e. transmission between two notes, 2) Ad-Hoc topology which is a dynamic setup of a network structure and 3) Hybrid topology or Mesh which is a combination of both [8], [10].

\section{SYSTEM ARCHITECTURE AND INITIAL DEVELOPMENT STEPS}

The proposed network that will be used to cover the patient's house will be based on motes like MicaZ ${ }^{\mathrm{TM}}$ while the acquisition of ECG data will be performed through a custom created board connected to the MDA300CA ${ }^{\mathrm{TM}}$ acquisition board. Additional environmental data will be collected through the MTS310CA ${ }^{\mathrm{TM}}$ sensor board. All collected information will be transmitted to a gateway, MIB510 ${ }^{\mathrm{TM}}$ connected on a Personal Computer; this is going to be the local monitoring system (see Figure 3). 


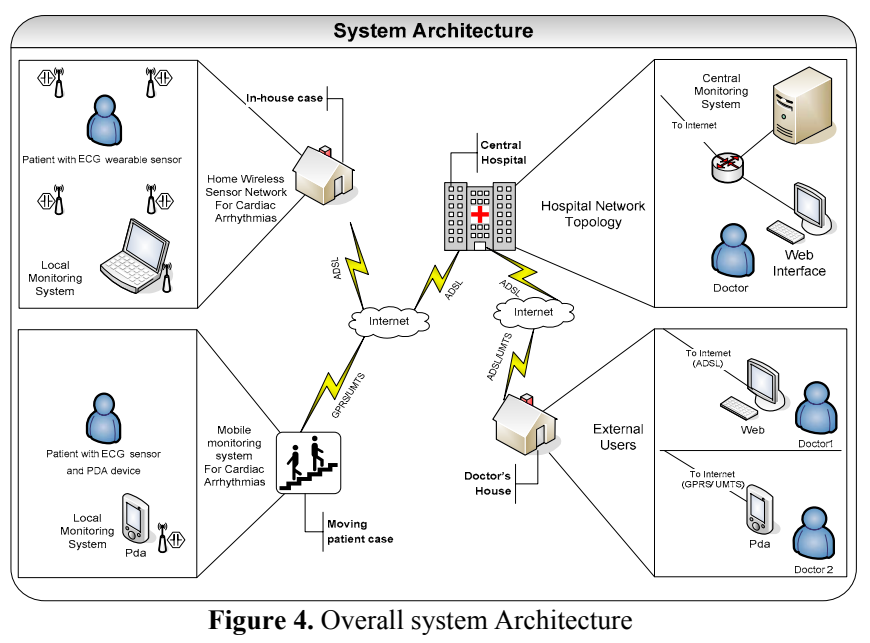

The in-house network will be connected to the remote monitoring system which is going to be responsible for reevaluating the transmitted signals, local storage of the signals and display on a web interface. It will be also responsible for the notification of the on-call doctor and a relative of the child. A similar scheme will be followed for the moving patient case, where ECG signals will be collected by the same ECG acquisition device and transmitted to the remote station through a mobile communication network. The remote station will perform the same actions as above. The overall system architecture diagram is shown in Figure 4.

Initial development steps have already been completed and a beta prototype is going through the test phase. The system is being developed using Microsoft Visual Studio(C) environment ( $\mathrm{C} \#$ and visual basic). An example of the web interface developed, displaying a test signal transmission, is shown in Figure 5. The display of the signal is divided into three sub-modules; the real time transmission of signals, the analysis module where ECG is presented with some analysis parameters based on the "PhysioNet Challenge" [11] and the 24 hour analysis which is displays summarized information about the last 24 hours of transmission.

\section{CONCLUDING REMARKS}

In this study, a prototype m-Health monitoring system for children with possible arrhythmias has been presented. As we have discussed, identification of children with arrhythmias is not an easy task and the treatment is not the same in all cases. Some forms of treatment, as is the case for some antiarrhythmia medications, increase the risk of arrhythmia (proarrhythmia effect) so it would be wrong to start such treatment without prior documentation of the problem. Another form of treatment uses implantable defibrillators. This form of treatment has given some promising results over the last few years. As we noted, it is difficult to decide who needs to be treated, not only because of the enormous cost but also because it entails a major

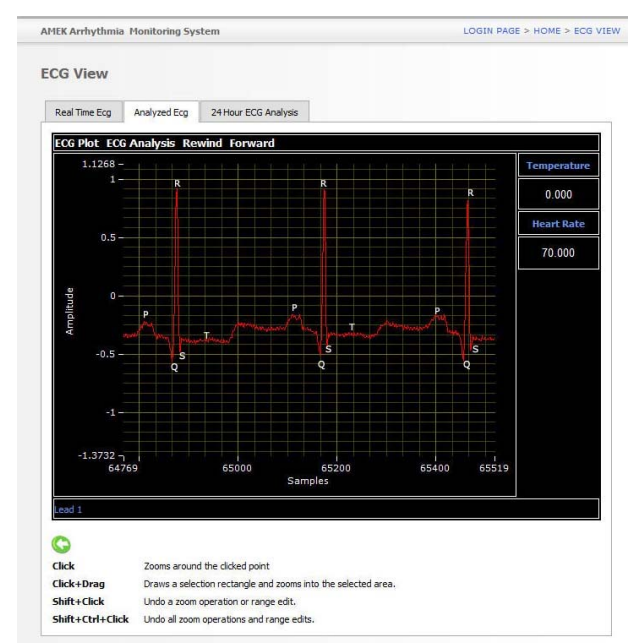

Figure 5. Server picture displaying the analyzed ECG

invasive procedure. It is clear that this special group of patients presents a unique and very difficult management problem amongst the various groups of patients that are at risk for arrhythmia. It is very important to monitor the ECG of these patients both in order to pick up the records that are at risk for arrhythmia and to record the type of arrhythmia that is needed before deciding on the proper management.

We hope that through the use of such a system we will be able to help in the identification of the type of problem thus helping the doctors provide the proper treatment. We have provided a basic architecture description and the initial development steps of the proposed system.

\section{REFERENCES}

[1] C.S. Pattichis et. al., "Wireless Telemedicine Systems: An Overview," IEEE Antennas \& Propag. Magazine, Vol. 44,No.2, pp143-153, 2002.

[2] F.C. Moreira et. al., "Noncompaction of the left ventricle: a new cardiomyopathy is presented to the clinician,"Sao Paulo Med. J., vol.124, No.1, pp.31-35, 2006.

[3] C. Stollberger, J. Finsterer, "Left ventricular hypertrabeculation /noncompaction," J. Am. Soc. Echocard., Vol.17,No.1,pp.91-100, 2004.

[4] E. Kyriacou, et.al., "Multi-purpose HealthCare Telemedicine Systems with mobile communication link support," BioMed.l Engin. OnLine, http://www.biomedical-engineering-online.com, Vol.2, No.7, 2003.

[5] R. Fensli, E. Gunnarson, T. Gundersen, "A wearable ECG-recording System for Continous Arrhythmia Monitoring in a Wireless TeleHome-Care Situation," in Proc. Of the $18^{\text {th }}$ IEEE Sypm. On Comp.Based Medical sys.(CBMSO5), 2005.

[6] V. Shnayder et al., "Sensor Networks for Medical Care," Harvard University Technical Report TR-08-05, April 2005.

[7] J. Proulux at. al. , "Development and Evaluation of a Bluetooth EKG Monitoring Sensor," in Proc. Of the $19^{\text {th }}$ IEEE Sypm. On Comp.-Based Medical sys.(CBMSO6), 2006.

[8] P. O. Bobbie, C. Deosthale, W. Thain, "Telemedicine: A Mote-Based Data Acqusition System for Real Time Health Monitoring," in proc. Telehealth 2006, Alberta, Canada, 2006.

[9] C. Nugent, et al., ECG TELECARE: Past Present and Future, in MHealth: Emerging Mobile Health Systems, Ed. By R. Istepanian, S. Laxminarayan, C.S.Pattichis, pp. 375-388, Springer, 2006.

[10] Crossbow Technology: Wireless Sensor Networks, Web page: http://www.xbow.com.

[11] F Canthi', et.al., "Predicting the End of an Atrial Fibrillation Episode: The PhysioNet Challenge," Computers in Cardiology, vol. 31, pp. 121-124, 2004. 